\title{
Dynamic Congestion Charging: Investigating Boundary Effects when Applying Low-Revenue Tolls
}

\author{
K. Stewart ${ }^{1}$ and Y. E. Ge ${ }^{2}$ \\ ${ }^{1}$ Transport Research Institute, Edinburgh Napier University, Merchiston Campus \\ Edinburgh, UK., EH10 5DT \\ ${ }^{2}$ School of Transportation \& Logistics, Dalian University of Technology, Dalian, \\ China \\ ${ }^{1}$ k.stewart@napier.ac.uk
}

\begin{abstract}
This paper investigates the impact of realistic congestion charging regimes where undesired boundary effects of imposing charges may occur and may be both temporal and spatial. Tolling regimes are developed with the primary aim to reduce system cost (total travel time) whilst also aiming to reduce undesired boundary effects. Two realistic toll profile regimes are investigated and tested; the first being flat tolls and the second bellshaped tolls which represent step-tolls and their relative impact on the boundary effects are discussed. This paper then investigates the reduction of travel time which may be achieved by applying different toll levels to each link and compares the cases where all links may be tolled against maintaining a single un-tolled route, which would be politically attractive. Existing DUE algorithms are utilised to obtain numerical results.
\end{abstract}

Keywords: Dynamic Traffic Assignment, Congestion Charging

\section{Introduction}

The classical road tolling problem in economics is to toll network links such that under the principles of Wardropian User Equilibrium (UE) assignment a System Optimizing (SO) flow pattern is obtained (where overall network benefit is maximized). Toll sets which induce the SO are non-unique, and further optimization is generally possible: for example, minimal revenue tolls create the desired SO flow pattern at minimal additional cost to the users, and generally allow a single path to remain un-charged. In the static case, the minimal revenue toll problem is capable of solution by various methods, such as linear programming (Bergendorff et al., 1997) and heuristically by reduction to a multicommodity max-flow problem (Dial, 2000). Previous work by Stewart (Stewart and Maher, 2006, Stewart, 2007) in a static stochastic environment has developed heuristics to determine low revenue tolls that produce good sub-optimal flow patterns where total network costs approach the system optimal. Extending to the dynamic case produces a large range of additional further objectives; primarily the tolls levied can vary with time and hence may be flat or profiled, there is usually a desired arrival-window imposed and tolling periods do not have to extend throughout the entire modelling period.

Dynamic Traffic Assignment (DTA) models extend the static concept of UE to DUE (dynamic user equilibrium) where the system is said to be in DUE where no user can unilaterally reduce their origin to destination travel time (or cost). Algorithms for DUE commonly iterate between two components; the Dynamic network loading (DNL) and route choice or path inflow reassignment (e.g., Wu et al., 1998, Lo and Szeto, 2002). It follows that DSO (Dynamic System Optimal) is where the total travel cost of all travellers through the network is minimised (over the time period under consideration), (Chow, 2007). Much work on DTA has focussed on whole-link models and consequently on DNL, recent work (Carey and $\mathrm{Ge}, 2011$ ) has concentrated on the routing aspect of dynamic 
modelling and has numerically compared various path-inflow reassignment methods by embedding them into a DUE algorithm and testing on simple network scenarios. Chow (2007) has presented a formulation for a dynamic system optimal but this is limited to a whole-link model rather than to a network model.

This paper utilizes existing DUE algorithms to investigate their ability to incorporate tolling scenarios and to produce low-revenue tolls to create desired flow profiles using network models. This paper investigates tolling strategies under different assumptions and determines toll sets which reduce (or minimize) total network cost under these assumptions. The variation of demand level whilst using a fixed toll has been examined (Stewart and Ge, 2010) for scenarios representing under congestion and saturated flow conditions. In all cases the feasibility of determining low-revenue toll sets to reduce (tending to minimize) the total network cost of a network under dynamic user equilibrium principles was demonstrated with the Total Network Cost surface as tolls varied being smooth and convex in the test networks examined (2-link and 4link/3path). In this paper toll profiles are both flat and bell-shaped (as per Ge and Stewart 2010b) the latter reducing the convergence issues from boundary effects and both spatial and temporal boundary effects will be discussed relative to the Path Inflow profiles across the time period. It will consider whether the objective of reducing aggregate TNTT to the maximum degree possible is a reasonable objective given a consideration of boundary issues and hyper-congestion (flow in excess of the maximum capacity) on network paths and links. Numerical results are presented examining firstly the no-toll flow profile and then the baseline effect of charging the entire time period (where spatial boundary effects do not occur). Results are then presented for scenarios using first fixed-tolls and then bellshaped tolls where different tolling subperiods are defined. Two tolling strategies are considered; minimizing Total Network Travel Time (TNTT) whilst leaving a single path uncharged and also where all paths may be charged.

\section{Model Formulation}

At present, the research on congestion charging in a time-varying context, has been investigated by means of the dynamic traffic assignment (DTA) technique. In the same manner as the general research on DTA, the research on dynamic congestion charging (DCC) has two streams. One focuses on the within-day scale and the other on the day-today scale. This paper will focus on within-day DCC and the congestion charging toll will be imposed on each path.

Figure 1 illustrates the DCC framework we have defined for use in this research; this is the same framework as that presented in Stewart and Ge (2010 and 2011). Each component of the framework is further discussed below.

\subsection{Tolling Scheme}

The tolling schemes considered in this research are path-based and all tolls are assumed to be collected at the entry of a path across the charging period.

Suppose that we consider a charging period $\left[t_{p}^{s}, t_{p}{ }^{e}\right]$, which is a subset of the time horizon $[0, T]$. Let $C_{p}{ }^{\text {toll }}(t)$ denote the toll (per vehicle) collected at the entry of path $p$ at time $t$, which satisfies the following condition;

$$
C_{p}^{\text {toll }}(t) \begin{cases}\geq 0 & \text { if } \quad t \in\left[t_{p}^{s}, t_{p}^{e}\right] \\ =0 & \text { otherwise }\end{cases}
$$

The charging period $\left[t_{p}^{s}, t_{p}^{e}\right]$ may vary in general from path to path, but in this paper the charging period will be the same on each of the three paths in the network. Previous papers examining low-revenue tolls (Stewart and Ge $(2010,2011)$ ) to reduce overall Total Network Travel Cost (TNTC) have examined the entire time [0,T], so as to avoid 
boundary issue complications; this paper however examines tolling solutions which have better network benefit than those presented in Ge and Stewart $(2010 \mathrm{~b}, 2012)$ and simultaneously examines the boundary issues of these relative to predetermined tolls.

The toll is only a part of the cost travellers consider in their trip decisions; in this paper the travel cost also includes schedule delay costs and travel time costs. We assume that there is a preferred arrival window, denoted as $\left[w^{l}, w^{r}\right]$. If a traveller arrives within the window $\left[w^{l}, w^{r}\right]$, there will be no schedule delay costs involved. Otherwise, a penalty will appear for early or late arrival at a destination. Denote the schedule delay cost of traffic entering path $p$ at time $t$ as $C_{p}(t+p(t))$, which depends on the entry time $t$ plus the travel time ${ }_{p}(t)$. Since the travel time ${ }_{p}(t)$. is the time traffic entering path $p$ at time $t$ would experience, $t+_{p}(t)$ is the time the traffic exits path $p$. We adopt the following widely used form of schedule delay costs in the literature

$$
C_{p}\left(t+\tau_{p}(t)\right)=\left\{\begin{array}{cc}
\beta\left[w^{l}-\left(t+\tau_{p}(t)\right)\right] & 0 \leq t+\tau_{p}(t)<w^{l} \\
0 & w^{l} \leq t+\tau_{p}(t)<w^{r} \\
\gamma\left[\left(t+\tau_{p}(t)\right)-w^{r}\right] & w^{r} \leq t+\tau_{p}(t) \leq T
\end{array}\right.
$$

where and respectively represent the shadow costs of early and late arrivals. As customary, they satisfy $<1$.

And then the generalized travel cost a traveller entering path $p$ at time $t$ would experience is

$$
\eta_{p}(t)=\alpha \tau_{p}(t)+C_{p}\left(t+\tau_{p}(t)\right)+C_{p}{ }^{\text {toll }}(t)
$$

in which the first term on the RHS of the equation represents the travel time cost, the second represents the schedule delay cost and the third is the congestion charging toll. The is the monetary value per unit time and satisfies, as customary, $<$. The values of these parameters may be dependent on origins or destinations. In this paper, they are assumed to be the same across the network under study. The travel time ${ }_{p}(t)$ is computed from the Network loading component, which will be described in Section 2.2.

The tolling schemes to be determined are to serve to fulfil the objective of reducing total network travel time in general and producing solutions which minimise the total network travel time subject to certain simplifying constraints. Thus we are not currently seeking to produce DSO tolls, but good suboptimal tolls based on practical constraints. Stewart and Ge (2010) has shown that it is feasible for fixed path tolls to produce a convex solution where the TNTC may be identified (under stated simplifying conditions), in practice however fixed tolls produce undesirable boundary peaks (Ge and Stewart $2010 \mathrm{a}, \mathrm{b}$ ) when introduced as a sub-period of the total charging period, which may be mitigated if a bell-shaped toll profile is utilised (which may be taken to represent steptolls). In this paper we consider the determination of path tolls which best reduce the TNTC across the network for two toll profile types: fixed and bell-shaped. 


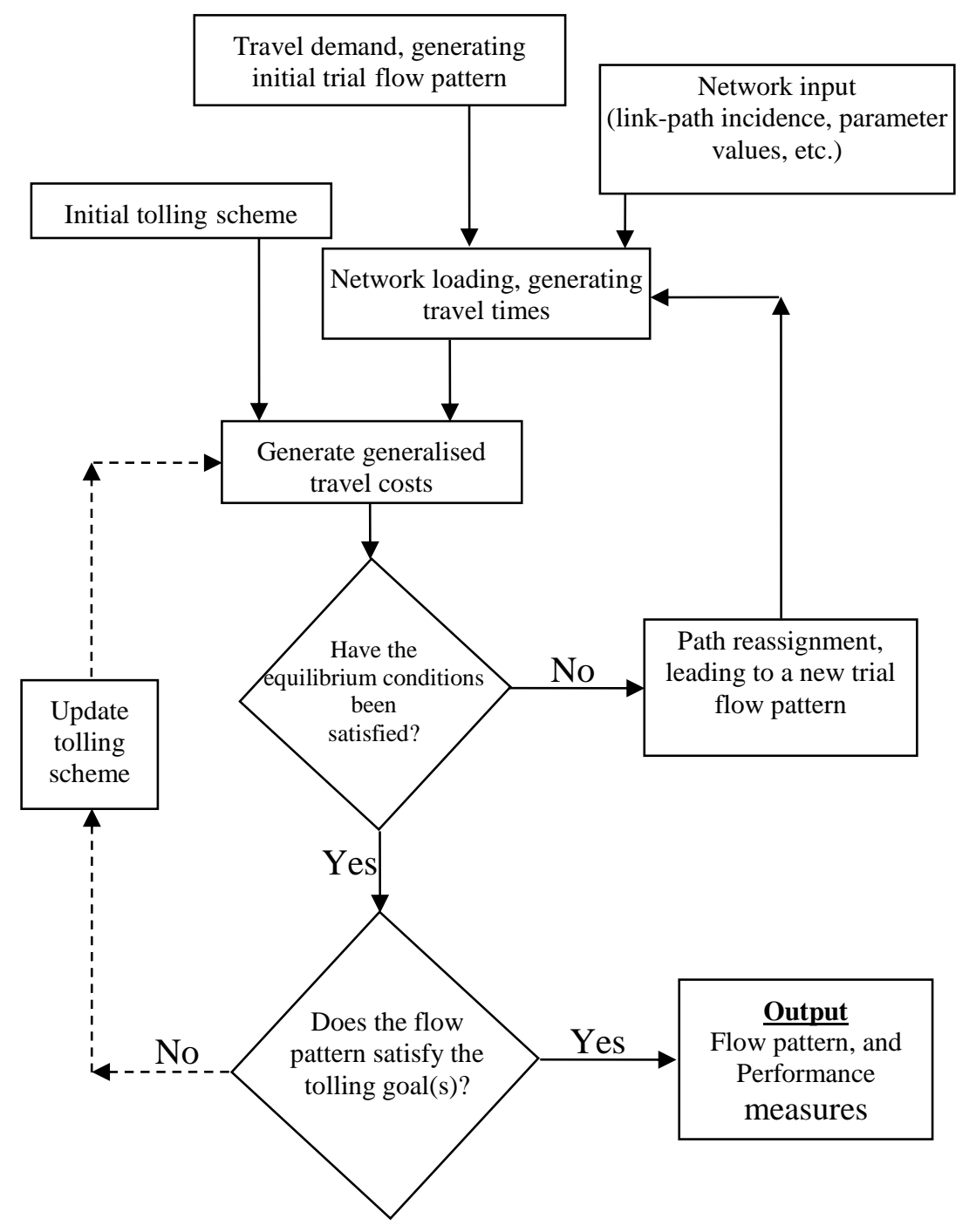

Figure 1. A Framework for Dynamic Congestion Charging

A further practical objective is to produce toll sets which do not impose full theoretical marginal costs upon the users, this is essentially the minimal revenue tolling principle familiar from static tolling literature (e.g., Dial, 2000). We take a heuristic approach to determine such low-revenue tolls as is given in previous work (e.g., Stewart and Maher, 2006, Stewart, 2007). Thus we always initialize our scheme with an initial zero-toll set, which presumes that we start from a current DUE solution based purely on the network costs. It is against the total travel cost from this initial DUE position that we will assess the effectiveness of our final tolling scheme.

When the DUE part of the algorithm is convergent, we then consider the tolling goals. We wish to produce tolls that minimise network cost given the tolling profile constraints that we have imposed for each scenario. We terminate the process when a toll increment on any of the paths serves to increase the total network cost from that achieved from a prior tolling scheme.

The update step, (as in the case of fixed tolls), relates to the relative toll level on each path. Firstly, a path hierarchy is generated, based on the relative benefit that tolling on a single path only could achieve. The path where the maximum reduction in total network 
cost could be achieved if tolling were permitted on a single link only is ranked first, and the remaining paths are ranked after this. The path which is ranked last should always remain un-tolled whilst following the low-revenue tolling objective, however in this paper we show that the TNTC may be reduced further if the final path is tolled incrementally in addition. This result holds under DTA when the toll-period is a sub-period of the entire period, and is not analogous to the static case where this feature is not applicable. . A toll increment is determined based on the accuracy of the solution required, and the tolls on the ranked paths are allowed to vary incrementally within a defined range.

\subsection{Network Loading}

In this paper, we will use a network loading procedure based on the cell-transmission technique. We implemented the procedure in the same way as in Daganzo (1995) and Lo and Szeto (2002). From this procedure, path travel times are obtained.

For Travel demand and initial trial flow patterns: it is assumed that the total travel demand over the time horizon for an origin-destination (OD) pair $w$ is $D^{w}$ and the demand at time $t$ for the OD pair is denoted as $g^{w}(t)$. Let $f_{p}(t)$ denote the flow rate entering path $p \in P^{w}$ at time $t$, where $P^{w}$ is a set of paths serving the OD pair $w . f_{p}(t)$ is subject to the traffic conservation condition and the non-negativity constraint below

$$
\begin{gathered}
\int_{0}^{T} g^{w}(t) d t=D^{w} \quad \forall w \\
\sum_{p \in P^{w}} f_{p}(t)=g^{w}(t) \quad \forall t \in[0, T], \forall w \\
f_{p}(t) \geq 0 \quad \forall t \in[0, T], \forall p \in P^{w}, \forall w
\end{gathered}
$$

If a flow pattern $f=\left\{f_{p}(t): \forall t \in[0, T], \forall p \in P^{w}, \forall w\right\}$ satisfies the above conditions, it is feasible.

An initial trial flow pattern should be feasible. In our numerical experiments, we assigned the demand $g^{w}(t)$ uniformly over the time horizon onto each path in $P w$. For Travel Time Generating: for each path $p$, the travel time ${ }_{p}(t)$ can be obtained by comparing the cumulative traffic on the entry and exit of it, represented by $Q_{p}(t)$ (entry) and $E_{p}(t)$ (exit), respectively. Specifically, the travel time at time $t$ is equal to the horizontal gap between $E_{p}(t+p(t))$ and $Q_{p}(t)$. A detailed description can be found in Lo and Szeto (2002) and Ge \& Carey (2004).

For Network input (link-path incidence, parameter values): we require the data for network loading, including the link-path incidence matrix, and free-flow speed, lengths, critical densities, jam densities and capacities for all links. Also, we need the data for $T$, the charging period, the time step size, etc. Parameters used in the numerical examples are given in Section 3.

\subsection{Path Reassignment}

Consider a single OD pair $w$. The path reassignment component determines a new trial flow pattern based on the travel costs $p(t)$. In this subsection, we will briefly look at the principle and methods for this purpose. Definition of equilibrium: before proceeding, we give the following definition of dynamic user equilibrium (DUE), which is an extension of the usual static user equilibrium to a dynamic context.

A path (in)flow pattern $\mathbf{f} \in \Omega=\left\{\mathrm{f}_{\mathrm{p}}(\mathrm{t}), \forall \mathrm{p} \in \mathrm{K}, \mathrm{t} \in[0, \mathrm{~T}] \mid \mathrm{f}_{\mathrm{p}}(\mathrm{t})\right.$ satisfies the conditions (4), $\left.\forall \mathrm{t} \in[0, \mathrm{~T}], \forall \mathrm{p} \in \mathrm{P}^{\mathrm{w}}, \forall \mathrm{w}, \mathrm{K}=\cup_{\mathrm{w}} \mathrm{P}^{\mathrm{w}}\right\}$ is a dynamic user equilibrium (DUE) solution if and only if, at any time $t$, the travel costs $\quad \mathrm{p}(\mathrm{t}), \forall \mathrm{p} \in \mathrm{K}$, is the same on all used paths over the time horizon and is less than on non-used paths. Thus , $\mathbf{f}^{*} \in \Omega$ is in DUE if and only if, at any time t, 


$$
\mathrm{p}(\mathrm{t})=\left\{\begin{array}{lll}
=\mu_{w}^{*} & \text { if } & f_{p}^{*}(t)>0 \\
\geq \mu_{w}^{*} & \text { if } & f_{p}^{*}(t)=0
\end{array} \forall \mathrm{p} \in \mathrm{P}^{\mathrm{w}}, \text { where } \mu_{w}^{*}=\min _{\mathrm{p}}\left\{\mathrm{p}(\mathrm{t}), \forall \mathrm{t} \in[0, \mathrm{~T}], \forall \mathrm{p} \in \mathrm{P}^{\mathrm{w}}\right\} .\right.
$$

for all OD pairs w. Friesz et al (2011) formulated the above DUE problem as an equivalent DVI (Differential Variational Inequality) problem and proposed a fixed-point algorithm to generate a new trial flow pattern based on the newly generated travel costs. Ge and Stewart (2010a) used this DVI model of the DUE problem and the fixed-point algorithm to investigate boundary issues arising from congestion charging. Here we utilise the DVI model and the algorithm and the computer code used for Ge \& Stewart (2010a).

\subsection{Toll Profiles}

The first type of toll profile (flat), defined as follows, allows the toll to stay at a constant level in the charging period $\left[t^{s}, t^{e}\right]$

$$
C_{\text {toll }}(t)= \begin{cases}u & t \in\left[t^{s}, t^{e}\right] \\ 0 & \text { otherwise }\end{cases}
$$

where the parameter $u$, representing the toll level, is a constant (in monetary unit), which also applies to the rest of the paper. (See Ge and Stewart 2010a, 2012 for slight smoothing approximation used in code to produce the above).

The $\underline{2}^{\text {nd }}$ type of toll profile (bell-shaped) is defined below:

$$
C_{\text {toll }}(t)=\left\{\begin{array}{cl}
u \sin \left(\frac{t-t^{s}}{t^{r}-t^{s}} \pi\right) & t \in\left[t^{r}, t^{s}\right] \\
0 & \text { otherwise }
\end{array}\right.
$$

\section{Scenario Settings for Numerical Tests}

This section set out the scenarios used for numerical analysis. One simple example network was used for all numerical experiments, having a single OD pair. Also, in each scenario the network is assumed to be empty at time $t=0$. The total travel demand is assumed to be fixed over the time horizon, specifically: $D=2156$ passenger units. In addition, the network is assumed to be empty at time $t=0$.At the initial iteration (i.e., determining the initial flow pattern), we assigned the OD travel demand equally over the time horizon among all paths for the OD pair. Also, if the inflow to a path in time interval $i$ is greater than the receiving capacity of its first link, or cell, then the excess traffic is held over to the next time interval, in which case there is a queue for entry to the first link of a path. Any waiting times of traffic for entry to a path were included in the corresponding path travel times. In all the analysis, the same time horizon $[0, T](T=35$ time units; (as for Ge and Stewart 2012) it may be assumed that 1 time unit $=6$ minutes, and hence a $3.5 \mathrm{hr}$ time period may represent 06.00-09.30). The same quadratic forms of flow-density $(q-k)$ relationships were used as in earlier papers and a network loading method based on the cell-transmission technique was used in this paper; for details see Ge and Stewart 2012.

The test network used has one OD pair and four links, as illustrated in Figure 2. The characteristics of the links are given in Table 1. It may be observed that the maximum flow rates on links 0 and 1 sum to $33.60=28.00=61.00$, which is greater than the capacity of the downstream link 2 and hence link 2 may act as a bottleneck under certain flow conditions. (For real-world comparison the flow $q_{l}{ }^{\max }$ in vehicle units/min, may be converted such that a vehicle unit $=5$ passenger cars and hence e.g., 33.60v.u. $/ \mathrm{min}=1680$ pcu/hr). Link 3 has a higher capacity than either link 0 or 1 , but lower than their sum. Since paths 1 and 2 (links 0,2 and 1,2) are shorter than path 3 (link 3) they will be chosen 
in preference to path 3 until congestion is such that path 3 is also chosen. The demand level has been set such that all paths are chosen in the modelled peak period. Throughout this paper the travellers' desired arrival window is fixed at $[25,30]$, with parameter vales; $\alpha=1.0, \beta=0.102, \gamma=0.4$ (eqns (2), (3)).

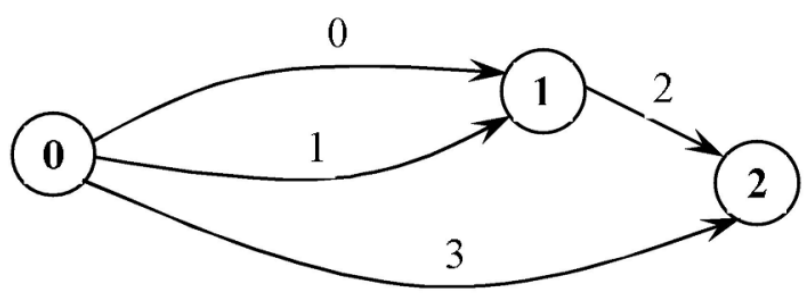

Figure 2. Example Network

Table 1. Characteristics of Four Links in Example Network (Figure 2)

\begin{tabular}{llllll}
\hline Link $l$ & $q_{l}^{\max }($ v.u./min $)$ & $v_{l}^{f}(\mathrm{~km} / \mathrm{min})$ & $L_{l}(\mathrm{~km})$ & $k_{l}^{c}(\mathrm{v} . \mathrm{u} . / \mathrm{km})$ & $k_{l}^{j}(\mathrm{v} . \mathrm{u} . / \mathrm{km})$ \\
\hline 0 & 33.60 & 1.200 & 1.20 & 56.00 & 160.00 \\
1 & 28.00 & 1.167 & 1.05 & 48.00 & 160.00 \\
2 & 57.50 & 1.250 & 0.50 & 92.0 & 280.00 \\
3 & 45.00 & 1.136 & 2.50 & 79.20 & 180.00 \\
\hline
\end{tabular}

\section{Scenario Results}

This paper considers the boundary effects resulting from the DCC scheme defined in Figure 1. The objective is to reduce the TNTC whilst maintaining the tolling objectives. In scenario 1 , the tolling sub-period is set to equal the entire period $[0,35]$, and in this case boundary temporal boundary effects should not be problematic. Introducing a fixed toll within the modelled time period will produce undesired boundary effects which may be reduced if a bell-shaped profile rather than a flat profile is used. In scenario 2 a flat profile is examined for tolling sub-period $[10,30]$ and $[5,30]$ and the tolls aiming to reduce TNTC are discussed in relation to fixed toll values of $45 \%$ and $25 \%$ of the no-toll DUE generalised cost. In scenario 3 the benefit of using bell-shaped tolls is examined against the same tolling sub-periods as in scenario 2. In each case the "minimal revenue" type toll solution where a single path remains uncharged is compared to a solution where all paths may be charged if TNTC may be reduced further.

\subsection{Baseline: Tolling sub-period is $[0,35]$-the Whole Modelled Period}

If the tolling period is the entire modelled period then it is clear that the model will not generate boundary effects. This short section is included as a reference baseline so that the ability of the tolling regimes to reduce network travel time may be observed prior to examining the boundary issues which form the main focus of the paper.

Previous work (Stewart and Ge 2011) examined the effect of altering the tolling subperiod when there was no arrival window (i.e., no arrival penalty). In this case, it was shown that altering the tolling sub-period had little or no benefit in terms of tolling being able to reduce TNTT. The same paper however, had shown that varying the arrival-time window could produce a range of feasible reductions in TNTT by tolling of $0.6 \%-11.4 \%$. In practice however, the arrival-time window and penalties for early or late arrival would be calibrated from data rather than being parameters which could be determined by policy makers. Consequently in this paper (as per Ge and Stewart 2012) we shall fix a predetermined arrival-time window of $[25,30]$ which represents a realistic arrival- 
window of $08.30-09.00$ in the AM peak. The parameters $\beta=0.102, \gamma=0.4$ for early and late arrival penalty remain fixed throughout this paper for clarity, but results have shown that these parameters are not unduly sensitive to perturbation.

As may be seen in Figure 3, the inflow on both paths 1 and 2 exceeds the maximum capacity on their first link for a time within the modelled period and hence congestion occurs, whilst path 3 is underutilised throughout the time period. It would be desirable to limit the congestion on paths 1 and 2 by tolling with path 3 remaining toll free.

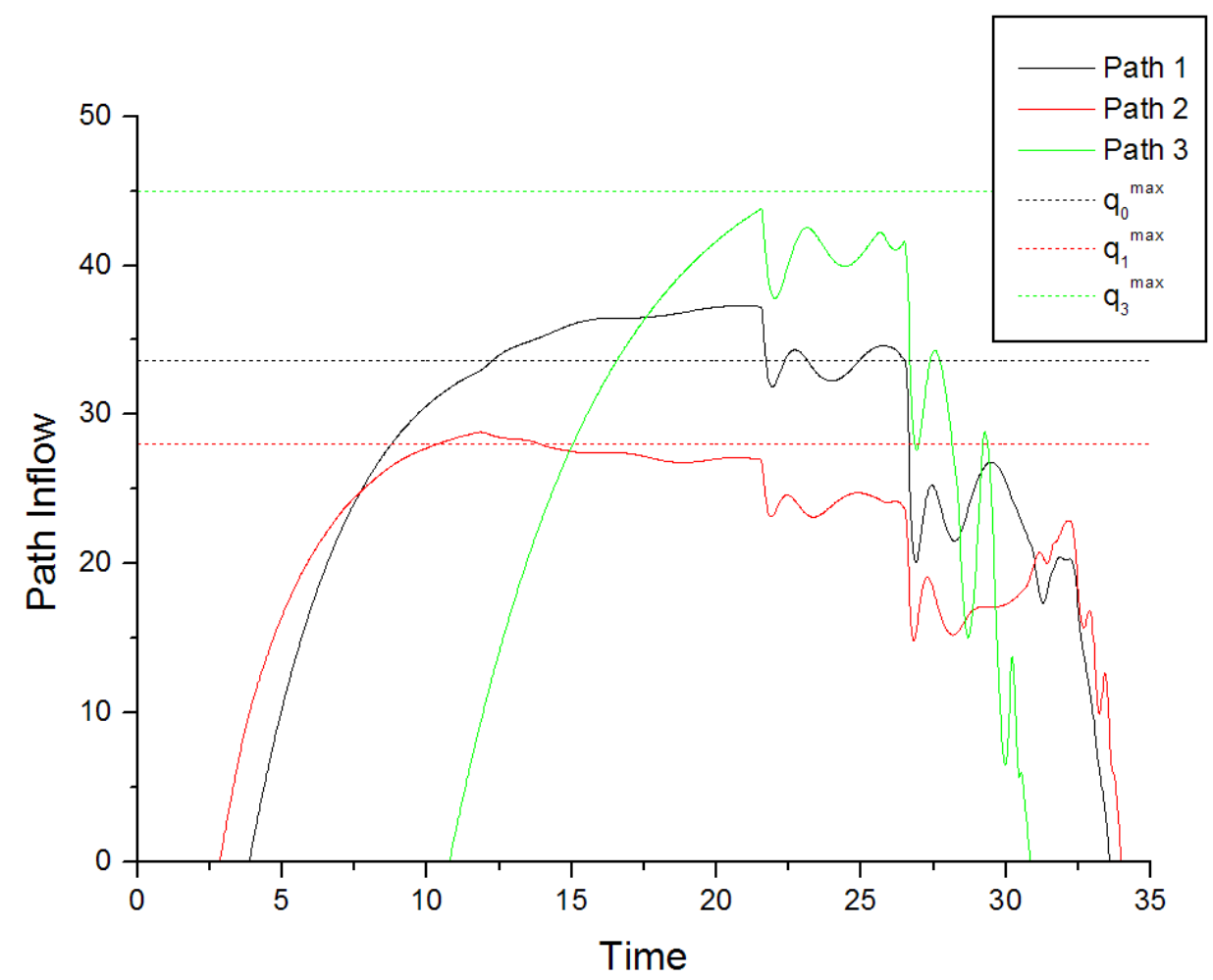

Figure 3. Path Inflow-no Tolls

Table 2. Toll-sets to Minimize TNTT

\begin{tabular}{lccccc}
\hline $\begin{array}{l}\text { Toll- } \\
\text { profile }\end{array}$ & $\begin{array}{c}\text { Toll-set } \\
\left(p_{1}, p_{2}, p_{3}\right)\end{array}$ & $\begin{array}{c}\text { Total Network } \\
\text { Travel time }\end{array}$ & $\begin{array}{c}\text { Total-toll } \\
\text { collected }\end{array}$ & $\begin{array}{c}\text { Reduction } \\
\text { in TNTT }\end{array}$ & $\begin{array}{c}\text { TNTT_Reduction } \\
\text { /1000mins(toll) }\end{array}$ \\
\hline Flat & $(0,0,0)$ & 6043.9 & 0 & $0 \%$ & - \\
Bell & $(1.0,1.1,0)$ & 5740.8 & 1085.4 & $5.0 \%$ & $4.6 \%$ \\
None & $(0,0,0)$ & 6043.9 & 0 & - & - \\
\hline
\end{tabular}

When the tolling sub-period is the full period $[0,35]$ it is not in fact possible to reduce the overall TNTT by use of a flat toll across the period - whilst some adjustment in path flows across the profile can occur these do not in this case result in network benefit. The use of the bell-shaped toll yields more favourable results with a feasible reduction in TNTT of 5\%. The Path-inflow profile which results is given in Figure 4. 


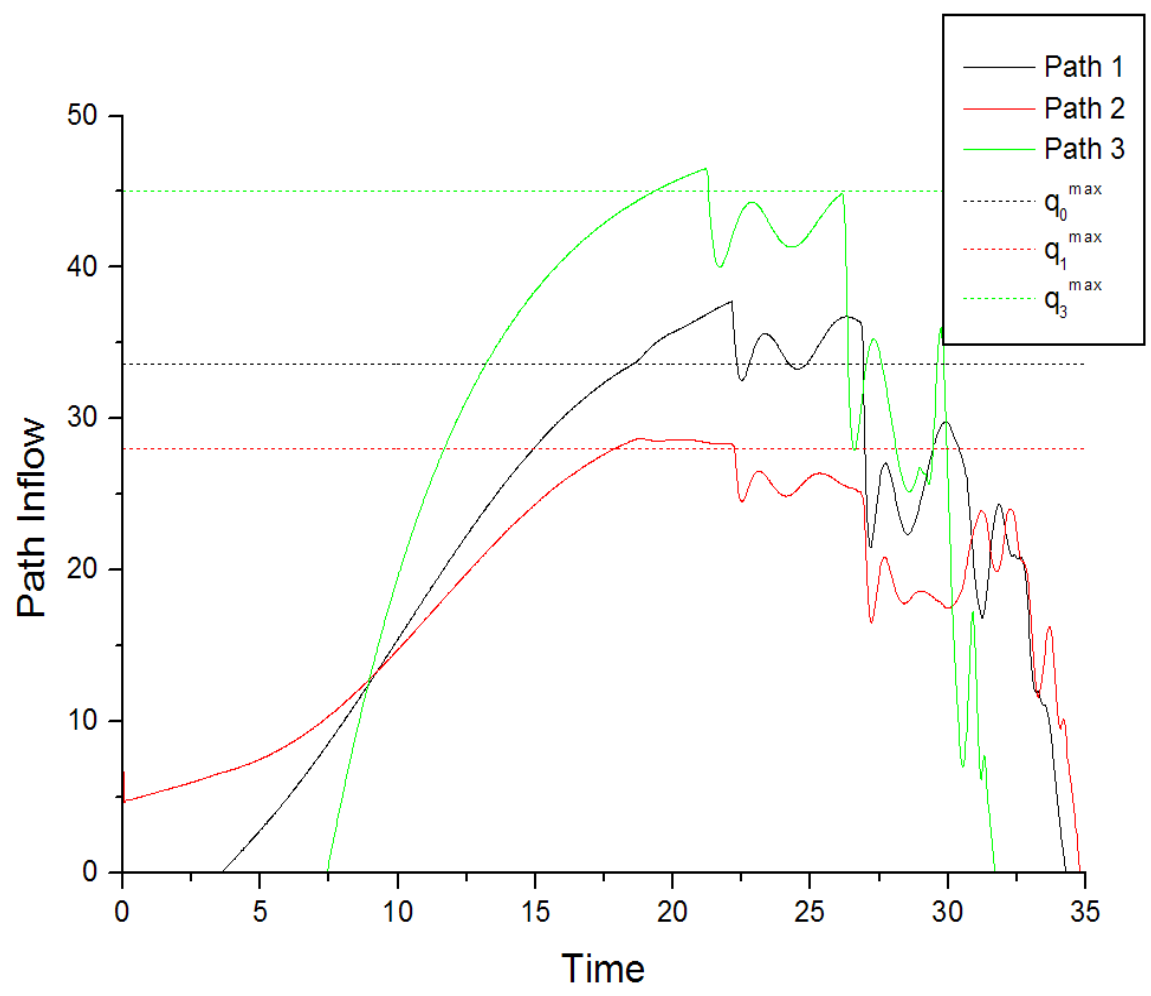

Figure 4. Path Inflow-Bell-tolls $(1.0,1.1,0)$

As may be observed, tolling the entire period does not result in boundary peaks, however it is not the case that all path-inflows remain strictly below the maximum capacity of their entry link. The duration for which flow on path 1 exceeds capacity is however reduced and the flows on the remaining paths meet (and slightly exceed) capacity for very short time periods. Path 3 is utilised more than in the no-toll case both in terms of total flow and in temporal terms for the length of time period where it is chosen. Further, congestion occurs later in the morning on all paths.

\subsection{Flat tolls-Tolling sub-period is $[10,30]$ and $[5,30]$}

Ge and Stewart (2012) have examined the boundary effects of introducing a flat-toll across the tolling sub- period [10,35] when the toll level is $45 \%$ and $25 \%$ of the no-toll DUE generalised cost. Equal tolls were levied on paths 1 and 2 and the toll levels resulting were $(1.539,1.539,0)$ and $(0.855,0.855,0)$. It was shown that both $45 \%$ and $25 \%$ toll levels reduced hyper-congestion but that undesired spatial and temporal boundary effects occurred, although these were greatly decreased by use of a lower toll. Here we wish to examine the use of tolls which are not necessarily equal on paths 1 and 2 , and which reduce the TNTT as far as is possible within the scenario specification. We examine two distinct toll-set solutions; the first where path 3 is to remain untolled to allow users to pick a "free" route option, the second is where the maximum reduction in TNTT across the network may be achieved and allows all 3-paths to be tolled. In scenario 1, where the tolling sub-period was the full modelled period, it was never possible to reduce aggregate TNTT by tolling path 3 . The existence of a "free" period before the beginning and after the end of the charging regime causes a possible network benefit from charging all paths. However equity issues should be considered, and the existence of uncharged routes may be a political objective which should be maintained. Here we consider both possible tolling solution types.

Table 3 illustrates the results with the shorter tolling sub-period of [10, 30] producing better absolute and relative network benefit for a lesser total toll imposed on the network 
than that for $[5,30]$. The greater ability for drivers to depart earlier to miss the start of the tolling period allows better aggregate reduction in TNTT.

Table 3. Flat-Toll Sets to Minimize TNTT with Tolling Sub-Periods

\begin{tabular}{lccccc}
\hline $\begin{array}{l}\text { Toll- } \\
\text { subperiod }\end{array}$ & $\begin{array}{c}\text { Toll-set } \\
\left(p_{1}, p_{2}, p_{3}\right)\end{array}$ & $\begin{array}{c}\text { Total Network } \\
\text { Travel time }\end{array}$ & $\begin{array}{c}\text { Total-toll } \\
\text { collected }\end{array}$ & $\begin{array}{c}\text { Reduction } \\
\text { in TNTT }\end{array}$ & $\begin{array}{c}\text { TNTT_Reduction } \\
\text { /1000mins(toll) }\end{array}$ \\
\hline$[10,30]$ & $(1.2,1.2,0)$ & 5402.8 & 1033.4 & $10.6 \%$ & $10.3 \%$ \\
{$[05,30]$} & $(1.2,1.1,0)$ & 5740.8 & 1129.2 & $5.9 \%$ & $5.2 \%$ \\
{$[10,30]$} & $(1.4,1.4,1.1)$ & 5065.8 & 1704.4 & $16.2 \%$ & $9.5 \%$ \\
{$[05,30]$} & $(1.3,1.2,1.1)$ & 5410.9 & 1939.7 & $10.5 \%$ & $5.4 \%$ \\
& $(0,0,0)$ & 6043.9 & 0 & - & - \\
\hline
\end{tabular}

Figure 5 shows the four path-inflow profiles for the optimising toll sets given above. Temporal boundary peaks may be observed at the beginning and end of the charging subperiod; when path 3 remains untolled undesired boundary effects are not observed on this path, but it is not chosen until later in the morning and is underutilised at some times. In all solutions large boundary peaks occur just after the end of the charging period, when drivers are wishing to enter the system as rapidly as possible to minimise their late arrival cost. It is possible that if the penalty for late arrival were adjusted then the additional toll would seem less undesirable and this boundary peak may be diminished. Where path 3 remains untolled it is more fully utilised with flows just reaching the maximum capacity in the middle of the time-period. Where path 3 is charged it becomes underutilised and contributes significantly to the toll-end boundary peak, in addition the flows on paths 1 and 2 exceed capacity more often when path 3 is tolled, so despite the network benefit in terms of TNTT, tolling all paths may be detrimental to traffic congestion at localised times.
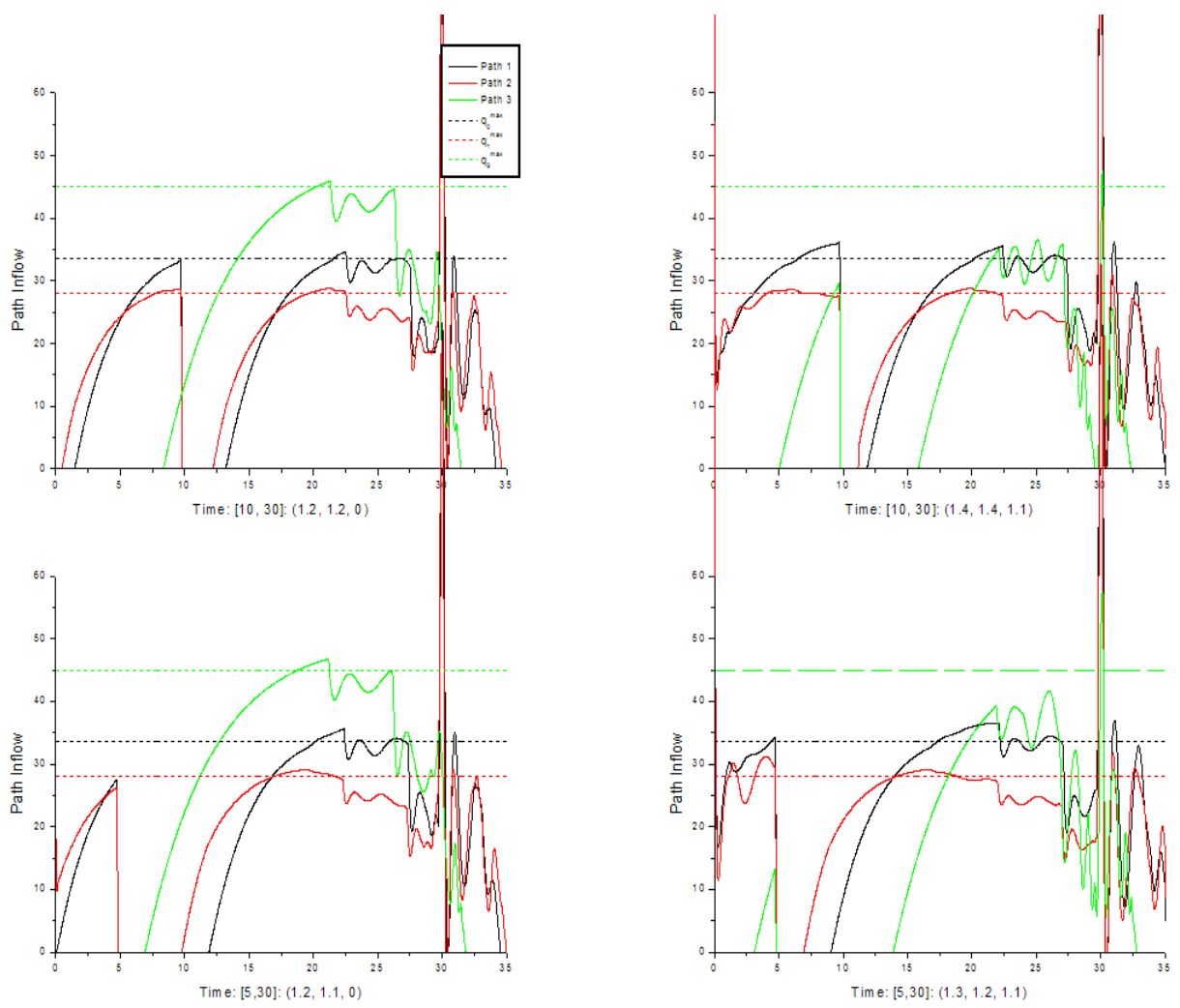

Figure 5. Path Inflow-Flat-tolls 


\subsection{Bell-shaped tolls-Tolling sub-period is $[10,30]$ and $[5,30]$}

Previous work (Ge and Stewart 2012) has suggested that tolls with a bell-shaped profile may mitigate adverse boundary effects. Here we apply the same principle for determining toll sets which reduce TNTT as in the fixed-toll case; the toll-value given for the bell-shaped-toll solutions is the maximum value of the toll profile. (See eqn (7)). Consequently, for the same toll-level presented, the total toll levied under bell-shaped tolls will be smaller than in the fixed-toll case, as lower tolls are levied either side of the maximum. As previously stated, the bell-shaped profile is intended to represent a "steptoll" which may be implemented in a realistic manner.

Table 4. Bell-Shaped-Toll Sets to Minimize TNTT with tolling sub-periods

\begin{tabular}{lccccc}
\hline $\begin{array}{l}\text { Toll- } \\
\text { subperiod }\end{array}$ & $\begin{array}{c}\text { Toll-set } \\
\left(p_{1}, p_{2}, p_{3}\right)\end{array}$ & $\begin{array}{c}\text { Total Network } \\
\text { Travel time }\end{array}$ & $\begin{array}{c}\text { Total-toll } \\
\text { collected }\end{array}$ & $\begin{array}{c}\text { Reduction } \\
\text { in TNTT }\end{array}$ & $\begin{array}{c}\text { TNTT_Reduction } \\
\text { /1000mins(toll) }\end{array}$ \\
\hline$[10,30]$ & $(1.3,1.4,0)$ & 5286.8 & 881.5 & $12.5 \%$ & $14.2 \%$ \\
{$[05,30]$} & $(1.0,1.1,0)$ & 5502.0 & 848.2 & $9.0 \%$ & $10.6 \%$ \\
{$[10,30]$} & $(1.6,1.7,1.0)$ & 5018.2 & 1351.7 & $17.0 \%$ & $12.6 \%$ \\
{$[05,30]$} & $(1.4,1.5,1.1)$ & 5243.2 & 1488.4 & $13.2 \%$ & $8.9 \%$ \\
& $(0,0,0)$ & 6043.9 & 0 & - & - \\
\hline
\end{tabular}

Table 4 presents equivalent results to those discussed in Table 3. The achievable reductions in TNTT are similar to those in the fixed case although slighter greater reduction in aggregate TNTT is achieved in all cases. For this reason alone it would appear that bell-shaped tolls (or step-tolls) would be preferable in practice to a fixed toll across the tolled sub-period.
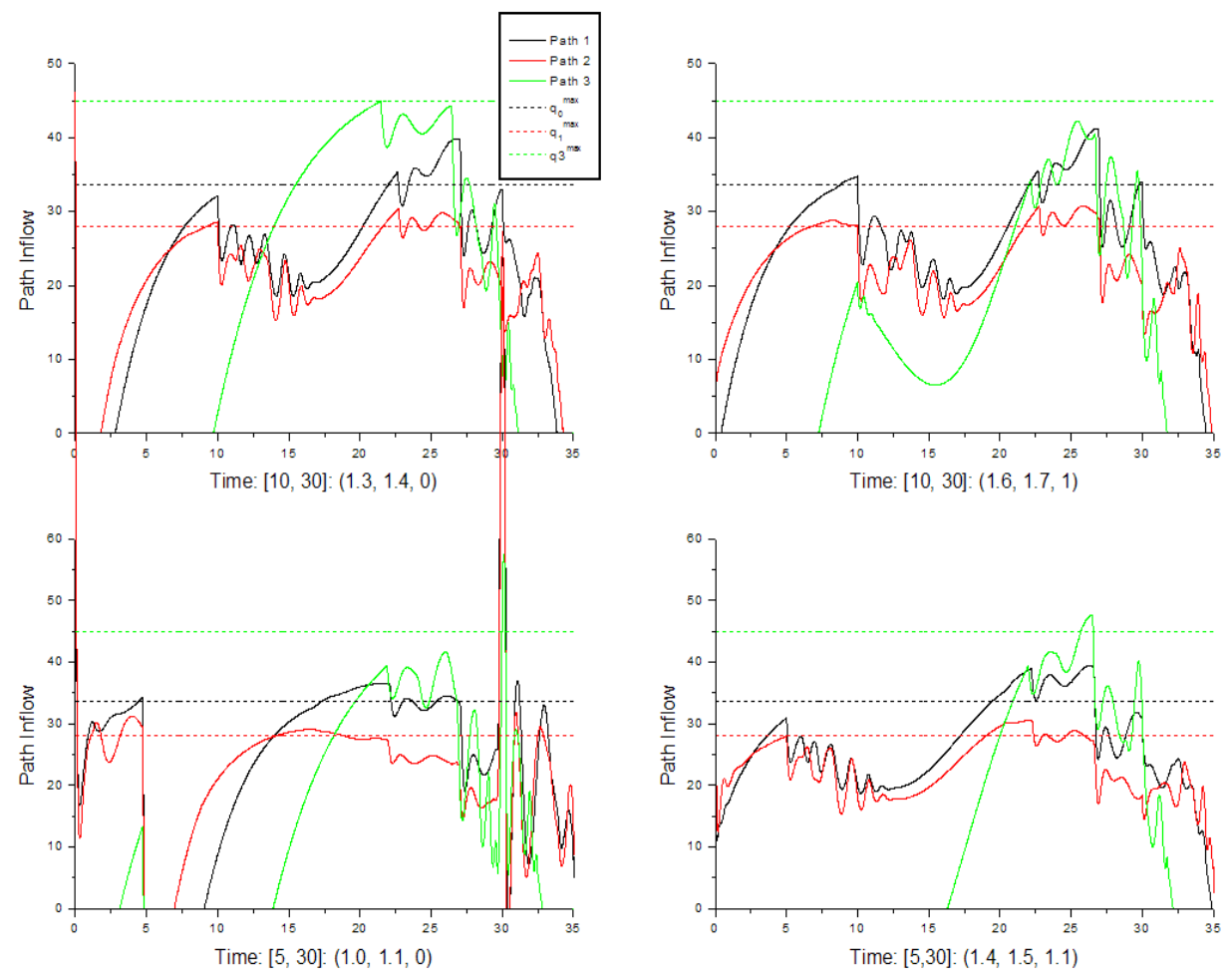

Figure 6. Path Inflow-Bell-Shaped-tolls 
The boundary effects may be observed in Figure 6. The undesirable high boundary peaks which were observed under fixed tolls at the end of the charging period appear to be almost eliminated by the bell-shaped tolls (except in one case). The tolled period [10, 30] where path 3 is uncharged appears favourable; path 3 is still underutilised before the charging period commences, at which point it becomes an attractive route. The traffic levels on path 3 do reach congestion point but do not exceed it, so whilst spatial boundary effects are being observed they are not such as to create hyper-congestion. Whilst tolling path 3 appears attractive from the aggregate network benefit perspective, it leaves path 3 significantly underutilised and produces more instances of hyper-congestion on path 1 than in the case where path 3 is untolled. The tolled period $[5,30]$ appears rather less favourable due (in likelihood) to the fewer opportunities for travellers to leave early to avoid the toll at the beginning of the charged period. Where path 3 is tolled, the path is again underutilised.

\section{Concluding Remarks}

This paper has examined the boundary effects across a modelled time-period when tolling regimes which seek to minimise TNTT (given scenario specifications) are imposed: two realistic toll-profile types were used; flat tolls across the charging period and bell-shaped tolls to represent step-tolling. For a baseline the paper first examined the case where tolls could be levied across the entire modelling period [0,35] which represents an AM period of 06.00-09.30. This (as expected) produced no boundary effects, however flat tolls were unable to reduce aggregate TNTT. Bell-shaped tolls did achieve moderate TNTT reduction but at the expense of producing significant congestion on path 3. In reality, tolls will be introduced for a sub-period of the entire day, so it is necessary to consider tolling sub-periods which lie within the modelled time horizon. In Sections 4.2 and 4.3 sub periods of $[10,30]$ (which represents charging between 07.00 and 09.00) and [5, 30] (which represents charging between 06.30 and 09.00) are considered for first flat-tolls and then bell-shaped. Two possible tolling regimes were examined; firstly where path 3 must remain untolled and secondly where all paths may be tolled to achieve maximum TNTT reduction. In the case where the entire period is tolled showed that there was never a benefit from tolling path 3; this is similar to results from the literature in static assignment where minimal revenue charging may (generally) be achieved with a path remaining toll-free. This feature is seen to be desirable as it promoted equity amongst drivers who may have a higher value of money than value of time, and who would prefer a longer, but untolled route. In the dynamic case however it is apparent that tolling all network paths will result in further aggregate benefit of TNTT reduction, however this policy may have adverse effects on boundary issues and underutilisation of the network in places at the expense of hyper-congestion in other locations. Political objectives must then be considered; is it preferable to reduce aggregate TNTT as much as is feasible, or should temporal issues of localised hyper-congestion be reduced?

It is clear that the boundary effects resulting from fixed tolls are more severe than for the bell-shaped tolls, substantiating the argument that step-tolls are more efficient that a single flat toll. It is further the case, that bell-shaped tolls (whether path 3 is tolled or not) produce better network benefit relative to flat-tolls. The charging sub-period should be determined to allow utilisation of all network paths by those drivers who wish to leave earlier or later than the charging period to avoid paying tolls; a longer untolled period relative to the desired arrival window appears more beneficial.

This paper has not adjusted the desired-arrival window as this factor is not generally one which may be altered by the operator, however the tolling sub-periods used have both coincided with the end of the arrival window. Further work will be undertaken to consider if the relative positions of the arrival window and tolling sub-period significantly impact 
on the temporal boundary issues observed, particularly those at the end of the charging period. The effect of adjusting the late arrival penalty will also be considered.

\section{References}

[1] P. Bergendorff, D. W. Hearn and M. V. Ramana, "Congestion Toll Pricing of Traffic Networks", Network Optimization, Pardalos P, Hearn D.W, Hager W.W, (Eds.), Lecture Notes in Economics and Mathematical Systems, Springer-Verlag, vol. 450, (1997), pp. 51-71.

[2] M. Carey and Y. E. Ge, "Comparison of methods for path inflow reassignment for dynamic user equilibrium", Networks and Spatial Economics, (2011).

[3] A. Chow, "Analysis of dynamic system optimum and externalities with departure time choice", Proceedings of Transportation and Traffic Theory, Eds Allsop, Bell, Heydecker, (2007), pp. 301-326.

[4] C. F. Daganzo, "The cell transmission model part II: network traffic", Transportation Research, vol. 29B, (1995), pp. 79-93.

[5] R. B. Dial, "Minimal-revenue congestion pricing part II: An efficient algorithm for the general case", Transportation Research, vol. 34B, no. 8, (2000), pp. 645-665.

[6] T. L. Friez, T. Kim, C. Kwon and M. A. Rigdon, "Approximate network loading and dual-time-scale dynamic user equilibrium", Transportation Research, vol. 45B, no. 1, (2011), pp. 176-207.

[7] Y. E. Ge and M. Carey, "Travel time computation of link and path flows and first-in-first-out", Proceedings of the 4th International Conference on Traffic and Transportation Studies, B. Mao, Z. Tian and Q. Sun (eds), Beijing: Science Press, (2004), pp. 326-335.

[8] Y. E. Ge and K. Stewart, "Investigating Spatial and Temporal undesired boundary effects arising from congestion charging", accepted for: DTA 2012; $4^{\text {th }}$ International Symposium on Dynamic Traffic Assignment, Martha's Vinyard, USA, (2012) June 4-6.

[9] Y. E. Ge and K. Stewart, "Investigating boundary issues arising from congestion charging in a bottleneck scenario", New Developments in Transport Planning: Advances in Dynamic Traffic Assignment, C. Tampère, F. Viti and L. H. Immers (eds), Publisher: Edward Elgar, (2010a), pp. 303326.

[10] Y. E. Ge and K. Stewart, "Investigating boundary issues arising from congestion charging, presented at the Fifth Travel Demand Management Symposium and revising for publication", Aberdeen, Scotland, U.K, (2010b) October 26-28.

[11] H. Lo and W. Y. Szeto, "A cell-based variational inequality of the dynamic user optimal assignment problem", Transportation Research Part B, vol. 36, (2002), pp. 421-443.

[12] K. Stewart, "Tolling traffic links under stochastic assignment: Modelling the relationship between the number and price level of tolled links and optimal traffic flows", Transportation Research A, vol. 41, no. 7, (2007), pp. 644-654.

[13] K. Stewart and Y. E. Ge, "Dynamic Congestion Charging: Optimising network flows with variable tolling periods", Szeto W.Y, Wong S.C, Sze N.N, (Eds.), Transportdynamics: Proceedings of the 16th International Conference of Hong Kong Society for Transportation Studies (HKSTS), ISBN: 978-98898847-9-6, (2011).

[14] K. Stewart and Y. E. Ge, "Optimising Network flows by Low-Revenue Tolling under the principles of Dynamic User Equilibrium", presented at the Fifth IMA International Mathematics in Transport Conference and revising for publication, UCL, London, U.K., (2010), April 12-14.

[15] K. Stewart and M. J. Maher, "Minimal revenue network tolling: system optimisation under stochastic assignment", Hearn D. W., Lawphongpanich S, Smith M, (Eds.), Mathematical and Computational Models for Congestion Charging, Applied Optimization, New York, Springer, vol. 101.

[16] J. H. Wu, Y. Chen and M. Florian, "The continuous dynamic network loading problem: a mathematical formulation and solution method”, Transportation Research Part B, vol. 32, (1998), pp. 173-187. 
International Journal of Transportation

Vol.3, No.2 (2015) 